\title{
Association between MTHFR C677T polymorphism and non-traumatic osteonecrosis of the femoral head: An update meta-analysis
}

https://doi.org/10.1515/pteridines-2020-0005

received August 24, 2019; accepted February 3, 2020.

\begin{abstract}
Objective To investigate the correlation between MTHFR C677T polymorphism and non-traumatic osteonecrosis of the femoral head. Methods Open published studies relevant to MTHFR C677T polymorphism and non-traumatic osteonecrosis of the femoral head were electronic systematic searched in the databases of cochrane central register of controlled trials, EMBSE and CNKI. The correlation between MTHFR C677T polymorphism and non-traumatic osteonecrosis of the femoral head was calculated by odds ratio (OR) and corresponding 95\% confidence interval $(95 \% \mathrm{CI})$. The publication bias for the included studies were assessed by Begg's funnel plot and Egger's line regression text. Results After systematic searching the electronic databases, 11 original studies were finally included the present work. The $\mathrm{I}^{2}$ test indicated significant statistical heterogeneity $\left(\mathrm{I}^{2}=53.5 \%, P=0.018\right)$ across the included 11 publications. The polled results indicated that subjects of Caucasians with CC genotype had decreased risk of developing non-traumatic osteonecrosis of the femoral head (OR=0.65,95\%CI: 0.44-0.96, $\mathrm{P}=0.031)$. However, there was no correlations between MTHFR C677T polymorphism and non-traumatic osteonecrosis of the femoral head in American Jewish and East Asian races $(\mathrm{p}>0.05)$. Sensitivity analysis indicated the pooled ORs were not sensitive to any included single study. The Begg's funnel plot was generally left and right symmetrical which indicated no
\end{abstract}

\footnotetext{
*Corresponding author: Zhenzhong Chen, Department of Spinal Surgery, Lishui Municipal Central Hospital, Zhejiang Province 323000 PR China, E-mail: chen37909@sina.com Tao Zhang, Department of Joint Surgery, Lishui Municipal Central Hospital, Zhejiang Province 323000 PR China

Yunmiao Ma, Department of orthopaedics, People's Hospital of Zhuji, Zhejiang Province, 311800 PR China

Shanshan Ye, Department of Pharmacy, Lishui Municipal Central Hospital, Zhejiang Province 323000 PR China

\# Equal contributor: Tao Zhang \& Shanshan Ye contributed equally to this work
}

obviously publications. The Egger's line regression test also demonstrated no statistical publication bias $(\mathrm{t}=1.57$, $\mathrm{P}=0.15$ ). Conclusion According to the present evidence, MTHFR C677T polymorphism was correlated with nontraumatic osteonecrosis of the femoral head especially for Caucasians race. Subjects of Caucasians race with CC genotype had decreased risk of developing non-traumatic osteonecrosis of the femoral head.

Keywords: MTHFR gene; non-traumatic osteonecrosis of the femoral head; polymorphism; meta-analysis.

\section{Introduction}

Non-traumatic osteonecrosis of the femoral head is a disease characterized by acquired ischemic lesions of the femoral head. Although globally epidemiological data are still lacking, some studies have shown that the highest incidence of the disease is in males aged 20 to 50 years [1-3]. As hip dysplasia and rapid progressive hip joint destruction, if the femoral head necrosis area reaches or exceeds $30 \%$ and remains untreated, nontraumatic osteonecrosis of the femoral head will develop into end-stage hip arthritis, which will lead to serious hip joint destruction, thus requiring surgical intervention. The etiology of non-traumatic osteonecrosis of the femoral head is not yet fully understood. Most studies indicated that the main pathophysiological mechanisms were excessive use of hormones and excessive intake of ethanol [3, 4]. Recently, several studies have evaluated correlations between some MTHFR gene polymorphism and susceptibility for non-traumatic osteonecrosis of the femoral head $[5,6]$. A meta-analysis included 8 trials published 7 years ago also indicated the correlation between MTHFR C677T polymorphism and non-traumatic osteonecrosis of the femoral head [7]. However, we found that this meta-analysis only included 8 studies and one of the included studies was about children, which may lead to significant clinical heterogeneity. Since 7 years had 
past, there were new studies published in the databases and may provide more evidence for the correlation between MTHFR C677T polymorphism and nontraumatic osteonecrosis of the femoral head. Therefore, we performed this up-date meta-analysis in order to provide more accurate information for the etiology of nontraumatic osteonecrosis of the femoral head

\section{Methods}

\section{Studies electronic searching strategy}

Open published studies relevant to MTHFR C677T polymorphism and non-traumatic osteonecrosis of the femoral head were electronic systematically searched in the databases of Cochrane central register of controlled trials, EMBSE and CNKI. The case-control or cohort clinical trials published in English or Chinese were systematic searched by the text words of: "MTHFR", "osteonecrosis of the femoral head", "Methylene tetrahydrofolate reductase", "polymorphism". The references of the included publications were also screened in order to further identify additional potential suitable publications that were not indexed in the electronic databases.

\section{Publication inclusion criteria}

Eligible publications should meet the below criteria: (1) Case-control or cohort designed clinical trials relevant to MTHFR C677T polymorphism and non-traumatic osteonecrosis of the femoral head. (2) Paper was published in English or Chinese; (3) Genotype frequency of both case and control groups can be extracted or calculated from the original studies; (4) The genotyping methods were correct; (5) There was no deviation of Hardy-Weinberg equilibrium (HWE) in the controls and case groups.

\section{Data extraction and evaluation}

Two reviewers (Yunmiao Ma \& Xiufeng Wang) independently review the included publications and extracted the data according to the Cochrane Handbook. If there was a disagreement of the data extraction, Two reviewers (Yunmiao Ma \& Xiufeng Wang) discuss the disagreement and consulted the third reviewer (Zhiyang Gao) to solve the problem. The general information of authors, year of the paper published, journals, ethnicity and genotyping methods of each included publication were extracted. The genotype frequency was carefully extracted or calculated by two reviewers from each included individual study independently and made crosschecking.

\section{Statistical analysis}

Stata/SE 11.0 (StataCorp LP, http://www.stata.com) statistical software was applied for data managing. The correlation between MTHFR C677T polymorphism and non-traumatic osteonecrosis of the femoral head was calculated by OR. Statistical heterogeneity among the included 11 studies was assessed through $\mathrm{I}^{2}$ test. Begg's funnel plot and Egger's line regression test were used for publication bias evaluation.

\section{Results}

\section{General character of the included 11 original publications}

After systematic searching, the electronic databases of Medline, the Cochrane central register of controlled trials, EMBSE and CNKI databases, 11 original studies [5, 6, 8-16] were finally included in the present work, Figure 1. Of the included 11 publications, 5 studies included the subjects of East Asian, 4 studies were about Caucasians and the other 2 were American Jewish. The general characteristics of the included 11 publications were shown in Table 1.

\section{Meta-analysis}

Before pooling the data, we first test the statistical heterogeneity across the included 11 studies by $\mathrm{I}^{2}$ test. The $\mathrm{I}^{2}$ test indicated significant statistical heterogeneity among the 11 publications $\left(\mathrm{I}^{2}=53.5 \%, P=0.018\right)$. Therefore, the data were pooled by random effect model. The pooled results indicated that subjects with CC genotype had decreased risk of developing non-traumatic osteonecrosis of the femoral head(OR=0.72,95\%CI:0.54-0.96, $\mathrm{P}=0.023$ ), Figure 2. 
Table 1: General information for the included 11 studies.

\begin{tabular}{|c|c|c|c|c|c|c|c|c|c|c|}
\hline First author & Year & Country & Ethnicity & Case & & & Contr & & & Methods \\
\hline & & & & $\mathrm{CC}$ & $\mathrm{CT}+\mathrm{TT}$ & Total & $\mathrm{CC}$ & $\mathrm{CT}+\mathrm{TT}$ & Total & \\
\hline Glueck & 1999 & U.S & American Jewish & 29 & 30 & 59 & 20 & 20 & 40 & PCR \\
\hline Glueck & 2002 & U.S & American Jewish & 10 & 26 & 36 & 120 & 115 & 235 & PCR \\
\hline Kutlar & 2001 & U.S & Caucasians & 29 & 16 & 45 & 54 & 8 & 62 & PCR \\
\hline Zalavras & 2002 & U.S & Caucasians & 22 & 44 & 66 & 117 & 183 & 300 & PCR \\
\hline Asano & 2004 & Japan & East Asian & 14 & 17 & 31 & 39 & 67 & 67 & PCR \\
\hline Liu & 2009 & China & East Asian & 13 & 76 & 89 & 18 & 59 & 77 & PCR \\
\hline Kim & 2010 & Korea & East Asian & 160 & 278 & 438 & 85 & 184 & 269 & PCR \\
\hline Chang & 2008 & Korea & East Asian & 18 & 53 & 71 & 80 & 120 & 200 & PCR \\
\hline Gagala & 2013 & Poland & Caucasians & 29 & 39 & 68 & 51 & 49 & 100 & PCR \\
\hline $\mathrm{Li}$ & 2015 & China & East Asian & 20 & 73 & 93 & 24 & 59 & 83 & PCR \\
\hline French & 2008 & U.S & Caucasians & 21 & 22 & 43 & 118 & 95 & 213 & PCR \\
\hline
\end{tabular}
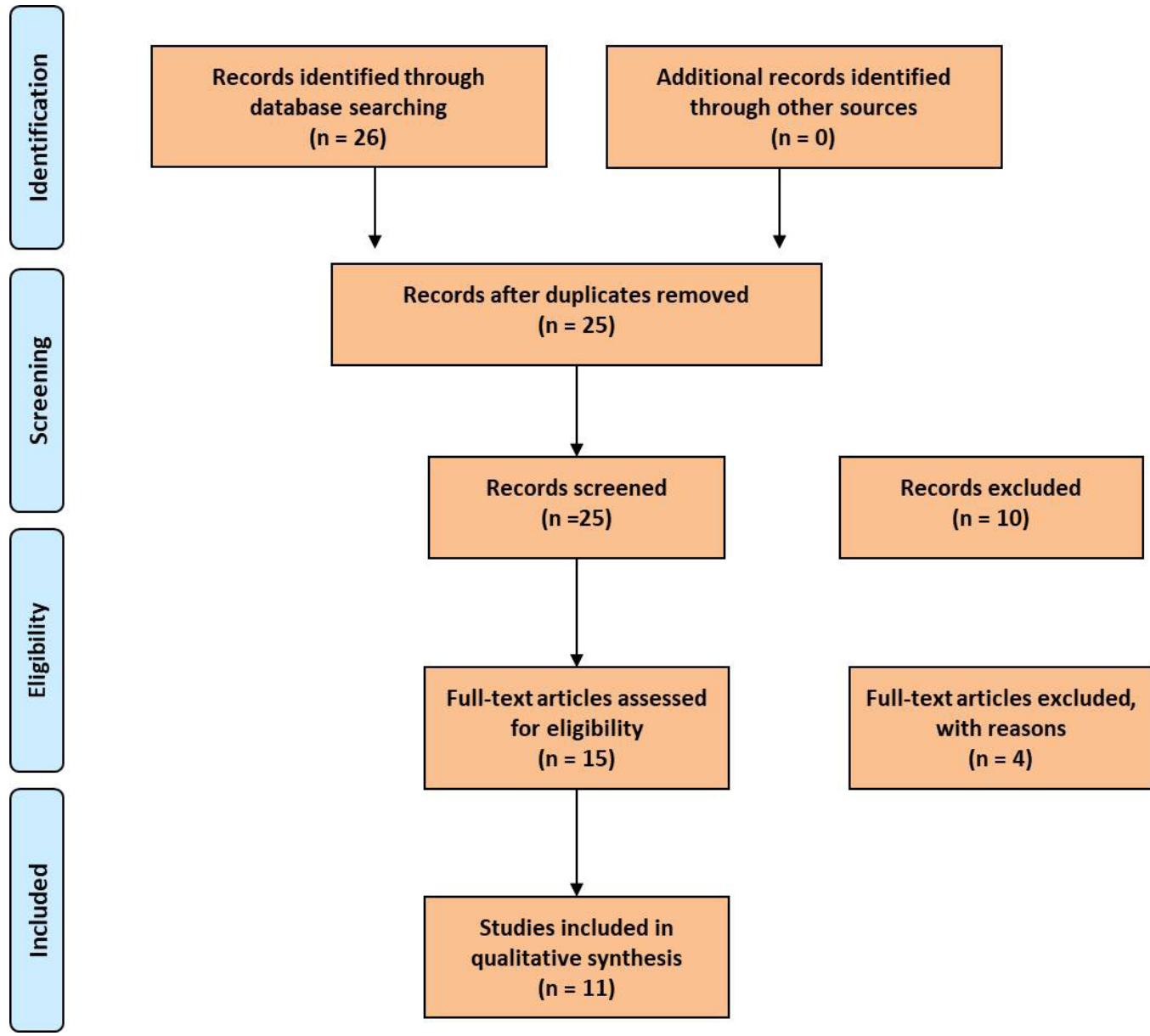

Figure 1: The publication searching flow chat of MTHFR C677T polymorphism and non-traumatic osteonecrosis of the femoral head. 
Study

ID

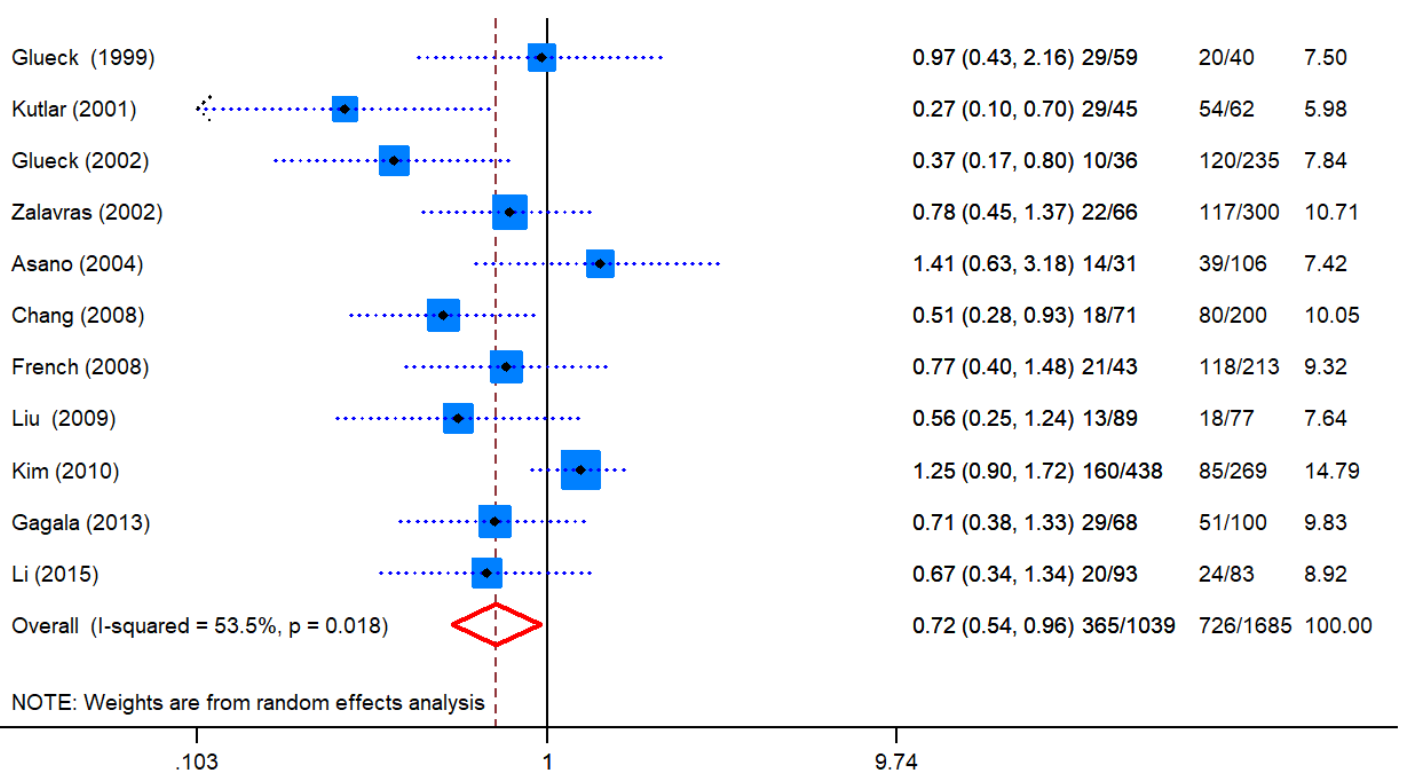

Figure 2: The forest plot of OR for MTHFR C677T polymorphism and non-traumatic osteonecrosis of the femoral head(CC vs CT+TT).

Study

ID



Figure 3. Subgroup analysis (American Jewish, Caucasians and East Asian ) of the forest plot of OR for MTHFR C677T polymorphism and nontraumatic osteonecrosis of the femoral head(CC vs CT+TT) 
Table 2: Subgroup analysis of MTHFR C677T polymorphism and nontraumatic osteonecrosis of the femoral head(CC vs CT+TT).

\begin{tabular}{lllll}
\hline Race & OR & $95 \% \mathrm{Cl}$ & p-value & Weight \\
\hline American Jewish & 0.59 & $0.23-1.53$ & 0.279 & $15.34 \%$ \\
Caucasians & 0.65 & $0.44-0.96$ & 0.031 & $35.84 \%$ \\
East Asian & 0.83 & $0.53-1.28$ & 0.399 & $48.83 \%$ \\
\hline
\end{tabular}

Table 3: The sensitivity analysis of MTHFR C677T polymorphism and non-traumatic osteonecrosis of the femoral head(CC vs CT+TT).

\begin{tabular}{llllll}
\hline $\begin{array}{l}\text { Study } \\
\text { omitted }\end{array}$ & Year & Estimate & $\begin{array}{l}\text { 95\% Confidence } \\
\text { interval }\end{array}$ & p-value \\
\hline \multicolumn{5}{c}{ Lower } & Upper \\
\hline Glueck & 1999 & 0.70 & 0.51 & 0.95 & 0.02 \\
Kutlar & 2001 & 0.77 & 0.59 & 1.00 & 0.05 \\
Glueck & 2001 & 0.77 & 0.58 & 1.00 & 0.05 \\
Zalavras & 2002 & 0.70 & 0.51 & 0.97 & 0.03 \\
Asano & 2004 & 0.68 & 0.51 & 0.91 & 0.01 \\
Chang & 2008 & 0.75 & 0.55 & 1.00 & 0.05 \\
French & 2008 & 0.71 & 0.52 & 0.97 & 0.04 \\
Liu & 2009 & 0.73 & 0.54 & 0.99 & 0.05 \\
Kim & 2010 & 0.66 & 0.51 & 0.84 & 0.01 \\
Gagala & 2013 & 0.71 & 0.52 & 0.98 & 0.04 \\
Li & 2015 & 0.72 & 0.52 & 0.98 & 0.04
\end{tabular}

\section{Subgroup analysis}

A subgroup analysis according to different races (American Jewish, Caucasians and East Asian ) was made Figure 3. The polled results indicated that subjects of Caucasians with CC genotype had decreased risk of developing non-traumatic osteonecrosis of the femoral head (OR=0.65,95\%CI: 0.44-0.96, $\mathrm{P}=0.031)$. However, there were no correlations between MTHFR C677T polymorphism and non-traumatic osteonecrosis of the femoral head in American Jewish and East Asian races ( $p>0.05)$, Table 2.

\section{Sensitivity analysis}

The sensitivity analysis of pooled results was performed by omitting each of the included studies, Figure 4. The results indicated the pooled ORs were not sensitive to any included single study, Table 3.

\section{Publication bias evaluation}

The publication bias in evaluation MTHFR C677T polymorphism and non-traumatic osteonecrosis of the femoral head were evaluated by Begg's funnel plot and Egger's line regression test. The Begg's funnel plot was generally left and right symmetrical which indicated no obviously publications, Figure 5. The Egger's line regression test also demonstrated no statistical publication bias $(\mathrm{t}=1.57, \mathrm{P}=0.15)$.

\section{Discussion}

Evidence has shown that intravascular coagulation and genetic factors are one of the pathogenesis of necrosis of the femoral head $[1,17]$. Homocysteine, the product of methylenetetrahydrofolate reductase gene, can promote the formation of thromboembolic lesions by damaging vascular endothelial cells and enhancing the coagulation activity of vascular [18]. The hyperhomocysteinemia caused by homocysteinemia is considered to be an independent risk factor for thrombosis. The polymorphism of methylenetetrahydrofolate reductase gene C677T locus has been confirmed to be associated with many ischemic diseases [7] and lung cancer [19]. In recent years, the relationship between the polymorphism of methylenetetrahydrofolate reductase gene C677T locus and the occurrence of femoral head necrosis has been widely discussed $[5,9]$.

The $\mathrm{C}>\mathrm{T}$ transition of methylenetetrahydrofolate reductase gene at nucleotide 677 may result in valine replacing highly conserved alanine in mature proteins, reducing enzyme activity and causing hyperhomocysteinemia. Homocysteine causes thromboembolic lesions by damaging vascular endothelial cells, proliferating vascular smooth muscle cells and enhancing the coagulation activity of vascular [20]. Therefore, this C>T SNP changes may increase the risk of developing necrosis of the femoral head.

A large number of studies have proved that the level of nitric oxide in atherosclerosis patients with methylenetetrahydrofolate reductase C677TT genotype is significantly reduced, while the high concentration of homocysteine can inactivate nitric oxide, resulting in vascular endothelial dysfunction [21-23]. Chang et al. [11] reported that 71 cases of femoral head necrosis were associated with methylenetetrahydrofolate reductase C677T polymorphism, of which 51 cases $(71.8 \%)$ were alcoholic femoral head necrosis patients. However, Asano et al. 


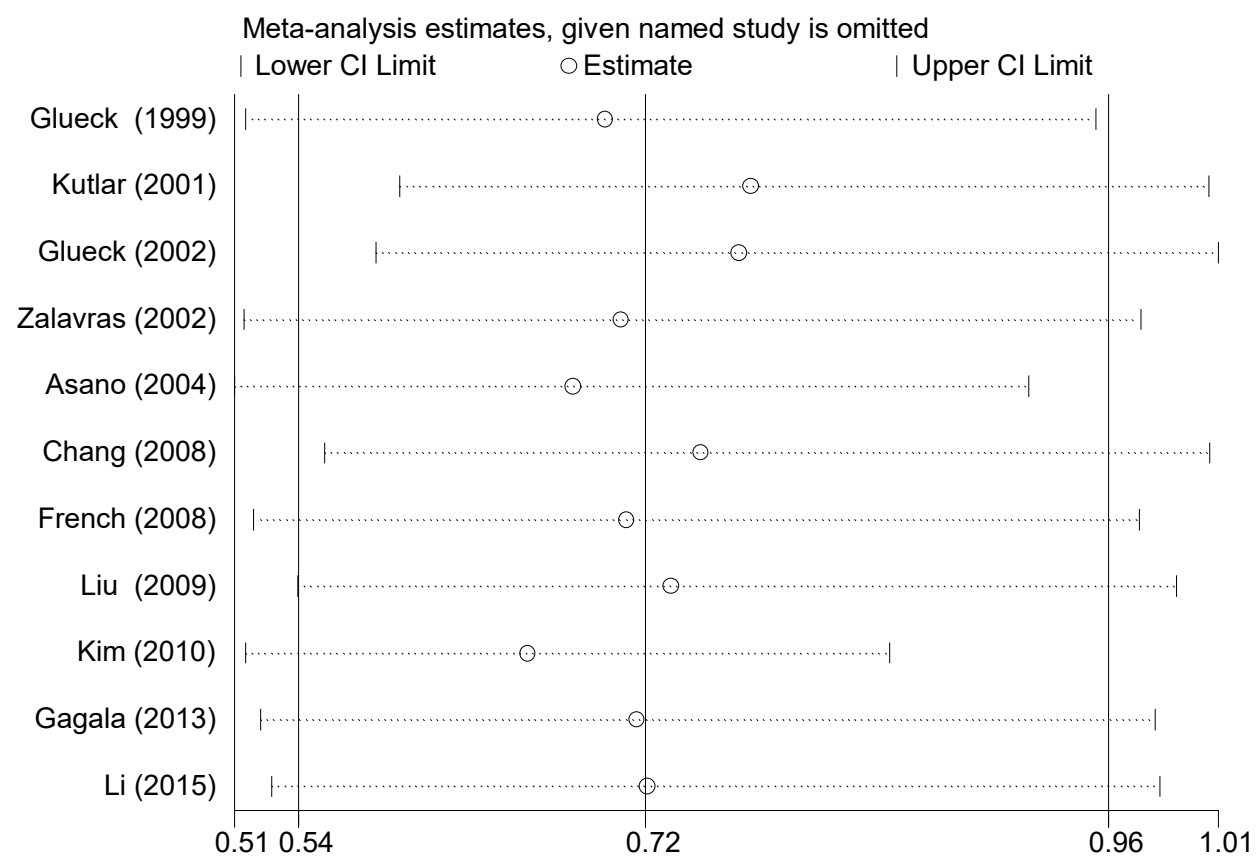

Figure 4: Sensitivity analysis of pooled results was performed by omitting each of the included study.

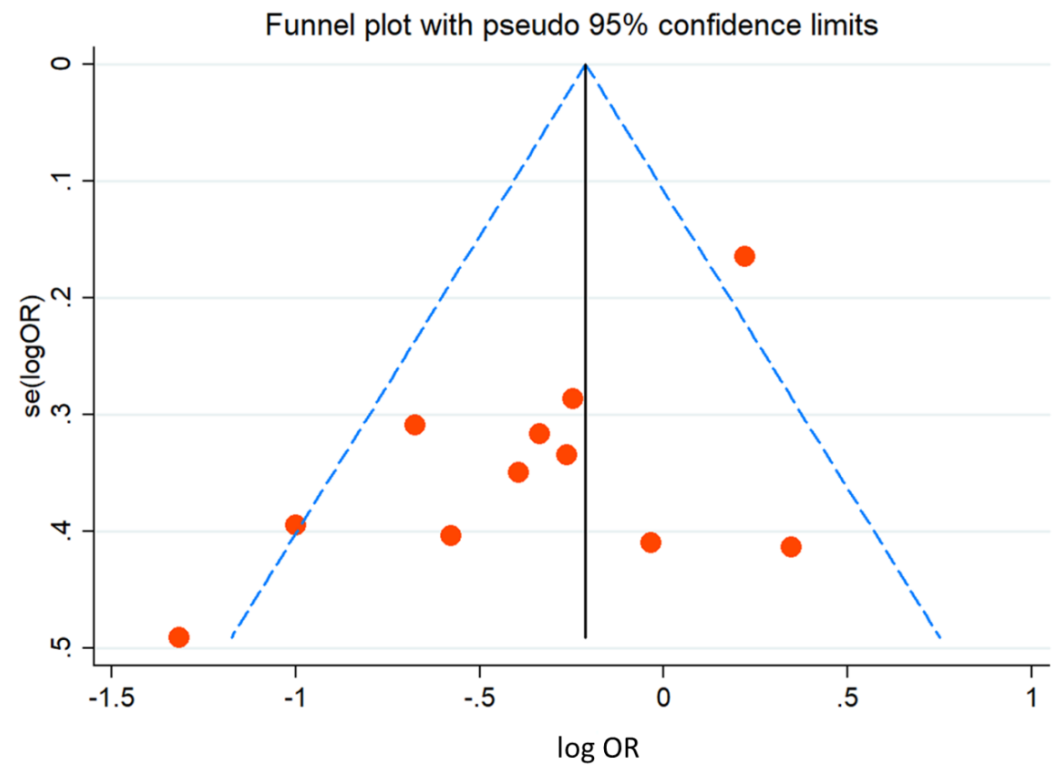

Figure 5: The Begg's funnel plot s indicated no significant publication bias in evaluation of MTHFR C677T polymorphism and non-traumatic osteonecrosis of the femoral head(CC vs CT+TT).

[6] reported that 31 patients with femoral head necrosis secondary to renal transplantation in Japan were not associated with methylenetetrahydrofolate reductase C677T polymorphism. A meta-analysis included 8 trials published 7 years ago also indicated the correlation between MTHFR C677T polymorphism and non-traumatic osteonecrosis of the femoral head. However, we found that this meta-analysis only included 8 studies and one of the included study was about children, which may lead to significant clinical heterogeneity. Therefore, the methylenetetrahydrofolate reductase C677T polymorphism is still controversial for the occurrence of femoral head necrosis. 
In our present work, we up-date the recently published case control studies relevant MTHFR C677T polymorphism and non-traumatic osteonecrosis of the femoral head and pooled the results. We found that MTHFR C677T polymorphism was correlated with non-traumatic osteonecrosis of the femoral head especially of the Caucasian's race. Subjects of the Caucasian's race with CC genotype had decreased risk of developing non-traumatic osteonecrosis of the femoral head. Subjects of Caucasians race with $\mathrm{CC}$ genotype had 0.65 risk of developing nontraumatic osteonecrosis of the femoral head compared to subjects of CT+TT genotype. And publication evaluation indicated no significant publication bias.

However, there were still some limitations. Firstly, in the present study, patients with femoral head necrosis were caused by different etiologies. This may a key potential clinical heterogeneity which may affect its general quality. Second, because folic acid and vitamin B12 both participate in the metabolism of homocysteine [24], folic acid and vitamin B12 concentrations can impact on homocysteine levels. Reduction of folic acid and vitamin B12 in the diet may cause hyperhomocysteinemia. So, the different folic acid and vitamin B12 intake from dietary may be another potential clinical bias. Third, significant statistical heterogeneity was also found among the included 11 studies. Therefore, better designed and largescale clinical studies are still needed to further evaluate the correlation between MTHFR C677T polymorphism and non-traumatic osteonecrosis of the femoral head. The interaction between genes and environmental factors should be further explored to provide a more comprehensive and reliable basis for the research and prevention of necrosis of the femoral head in order to alleviate the disease.

Conflict of interest: Authors state no conflict of interest.

\section{References}

1. Petek D, Hannouche D, Suva D. Osteonecrosis of the femoral head: pathophysiology and current concepts of treatment. EFORT Open Rev. 2019 Mar;4(3):85-97.

2. Cohen-Rosenblum A, Cui Q. Osteonecrosis of the Femoral Head. Orthop Clin North Am. 2019 Apr;50(2):139-49.

3. Baig SA, Baig MN. Osteonecrosis of the Femoral Head: Etiology, Investigations, and Management. Cureus. 2018 Aug;10(8):e3171.

4. Malizos KN, Karantanas AH, Varitimidis SE, Dailiana ZH, Bargiotas K, Maris T. Osteonecrosis of the femoral head: etiology, imaging and treatment. Eur J Radiol. 2007 Jul;63(1):16-28.
5. Kutlar A, Kutlar F, Turker I, Tural C. The methylene tetrahydrofolate reductase (C677T) mutation as a potential risk factor for avascular necrosis in sickle cell disease. Hemoglobin. 2001 May;25(2):213-7.

6. Asano T, Takahashi KA, Fujioka M, Inoue S, Ueshima K, Hirata T, et al. Relationship between postrenal transplant osteonecrosis of the femoral head and gene polymorphisms related to the coagulation and fibrinolytic systems in Japanese subjects. Transplantation. 2004 Jan;77(2):220-5.

7. Shang XF, Su H, Chang WW, Wang CC, Han Q, Xu ZW. Association between MTHFR C677T polymorphism and osteonecrosis of the femoral head: a meta-analysis. Mol Biol Rep. 2012 Jun;39(6):7089-94.

8. Glueck CJ, Fontaine RN, Gruppo R, Stroop D, Sieve-Smith L, Tracy $\mathrm{T}$, et al. The plasminogen activator inhibitor-1 gene, hypofibrinolysis, and osteonecrosis. Clin Orthop Relat Res. 1999 Sep;366:133-46.

9. Glueck CJ, Freiberg RA, Fontaine RN, Tracy T, Wang P. Hypofibrinolysis, thrombophilia, osteonecrosis. Clin Orthop Relat Res. 2001 May;386:19-33.

10. Li S, Zhou M, Guo T, Wang X, Sun F, Mu H. Relation study between TCM constitution types of nontraumatic osteonecrosis of femoral head and polymorphism of methylene tetrahydrofolate reductase $\mathrm{C} 677 \mathrm{~T}$ site. Zhonghua Zhongyiyao Zazhi. 2015; 2194-8.

11. Chang JD, Hur M, Lee SS, Yoo JH, Lee KM. Genetic background of nontraumatic osteonecrosis of the femoral head in the Korean population. Clin Orthop Relat Res. 2008 May;466(5):1041-6.

12. Zalavras CG, Vartholomatos G, Dokou E, Malizos KN, Factor V. Factor $\mathrm{V}$ Leiden and prothrombin gene mutations in femoral head osteonecrosis. Thromb Haemost. 2002 Jun;87(6):1079_ 80.

13. Liu B, Li Z, Sun W, Wang B, Shi S, Min H. [Relationship between alcohol induced osteonecrosis of femoral head and single nucleotide polymorphisms of methylene tetrahydrofolate reductase 677 C/T]. Zhongguo Xiu Fu Chong Jian Wai Ke Za Zhi. 2009 Sep;23(9):1079-82.

14. Kim TH, Hong JM, Kim HJ, Park EK, Kim SY. Lack of association of MTHFR gene polymorphisms with the risk of osteonecrosis of the femoral head in a Korean population. Mol Cells. 2010 Apr;29(4):343-8.

15. Gagala J, Buraczynska M, Mazurkiewicz T, Ksiazek A. Prevalence of genetic risk factors related with thrombophilia and hypofibrinolysis in patients with osteonecrosis of the femoral head in Poland. BMC Musculoskelet Disord. 2013 Sep;14(1):264.

16. French D, Hamilton LH, Mattano LA Jr, Sather HN, Devidas M, Nachman JB, et al.; Children's Oncology Group. A PAI-1 (SERPINE1) polymorphism predicts osteonecrosis in children with acute lymphoblastic leukemia: a report from the Children's Oncology Group. Blood. 2008 May;111(9):4496-9.

17. Moya-Angeler J, Gianakos AL, Villa JC, Ni A, Lane JM. Current concepts on osteonecrosis of the femoral head. World J Orthop. 2015 Sep;6(8):590-601.

18. Matsuo K, Hirohata T, Sugioka Y, Ikeda M, Fukuda A. Influence of alcohol intake, cigarette smoking, and occupational status on idiopathic osteonecrosis of the femoral head. Clin Orthop Relat Res. 1988 Sep;\&NA;(234):115-23. 
19. Fang Liu GQ, Tingyu Tang QH, Zhijun Li HH, Lu X.

Methylenetetrahydrofolate Reductase (MTHFR) Gene rs1801133

C>T Polymorphisms and Lung Cancer Susceptibility: An Updated Meta-analysis. Pteridines. 2019;30(1):65-73.

20. Mont MA, Zywiel MG, Marker DR, McGrath MS, Delanois RE. The natural history of untreated asymptomatic osteonecrosis of the femoral head: a systematic literature review. J Bone Joint Surg Am. 2010 Sep;92(12):2165-70.

21. Gardemann A, Weidemann H, Philipp M, Katz N, Tillmanns H, Hehrlein FW, et al. The TT genotype of the methylenetetrahydrofolate reductase $\mathrm{C677T}$ gene polymorphism is associated with the extent of coronary atherosclerosis in patients at high risk for coronary artery disease. Eur Heart J. 1999 Apr;20(8):584-92.

22. Bova I, Chapman J, Sylantiev C, Korczyn AD, Bornstein NM. The A677V methylenetetrahydrofolate reductase gene polymorphism and carotid atherosclerosis. Stroke. 1999 Oct;30(10):2180-2.

23. Lim PS, Hung WR, Wei YH. Polymorphism in methylenetetrahydrofolate reductase gene: its impact on plasma homocysteine levels and carotid atherosclerosis in ESRD patients receiving hemodialysis. Nephron. 2001 Mar;87(3):249-56.

24. Ueland PM, Hustad S, Schneede J, Refsum H, Vollset SE. Biological and clinical implications of the MTHFR C677T polymorphism. Trends Pharmacol Sci. 2001 Apr;22(4):195-201. 